\title{
DEFECTS FOR RANDOM TELEGRAPH NOISE AND NEGATIVE BIAS TEMPERATURE INSTABILITY
}

\author{
J. F. Zhang, M. Duan, Z. Ji, and W. Zhang \\ Department of Electronics and Electrical Engineering, Liverpool John Moores University, \\ Byrom Street, Liverpool L3 3AF, UK \\ E-mail: j.f.zhang@1jmu.ac.uk
}

\begin{abstract}
Random Telegraphy Noise (RTN) and Negative Bias Temperature Instability (NBTI) are two important sources of device instability. Their relation is not fully understood and is investigated in this work. We examine the similarity and differences of the defects responsible for them. By following the As-grown-Generation (AG) model proposed by our group, we present clear evidences that the As-grown hole traps (AHTs) are responsible for the RTN of pMOSFETs. AHTs also dominate NBTI initially, but the generated defects (GDs) become increasingly important for NBTI as stress time increases. The GDs, however, do not cause RTN.
\end{abstract}

\section{INTRODUCTION}

As device sizes are downscaled to the nanometer range, both Random Telegraphy Noise (RTN) and Negative Bias Temperature Instability (NBTI) can adversely affect the pMOSFET and, in turn, circuit performance and they have attracted a lot of attentions [112]. The relationship between RTN and NBTI is not fully understood, although it was suggested that they are the two facets of the same mechanism [1]. This lack of understanding is because the similarity and differences of the defects responsible for RTN and NBTI are not fully understood yet.

It is generally accepted that positive charging and discharging in the gate dielectric are responsible for RTN and NBTI [1-3]. The positive charges, however, behave in a complex way and have caused confusions since 1970's [4,5]. Recently, we proposed an As-grownGeneration (AG) model for positive charges in gate dielectric [3,6-8]. In this work, we will use this framework to examine the defects responsible for RTN and NBTI and through it, clarify their relation.

\section{DEVICES AND EXPERIMENTS}

The pMOSFETs have a TiN gate, a channel length of $50 \sim 10,000 \mathrm{~nm}$, and a width of 90 10,000 nm. The gate dielectric stack is $\mathrm{SiON} / \mathrm{HfO}_{2}$ and the equivalent oxide thickness is $1.45 \mathrm{~nm}$.

To ensure capturing all defects, RTN was measured with a sampling rate of $10 \mathrm{M} / \mathrm{sec}$ [9]. To avoid recovery during NBTI measurements, the fast pulse technique has been applied with a measurement time of $5 \mu \mathrm{s}$ [10]. All tests were carried out at $125^{\circ} \mathrm{C}$.

\section{AS-GROWN-GENERATION (AG) MODEL}

The AG model divides the positive charges formed in the gate dielectric into two groups: as-grown hole traps (AHTs) and generated defects (GDs) [3,6-8]. They can be separated from their different energy locations. By using the energy profile technique developed recently [11], it has been shown that the AHTs are below the silicon valence band edge, Ev, whilst the GDs are above it, as illustrated by Fig. 1(a).

To support this separation, Fig. 1(b) shows that the defects above Ev increases with stress time, because of the generation of new defects. The defects below Ev, however, remain the same, as represented by the two double arrowed vertical lines in Fig. 1(b).

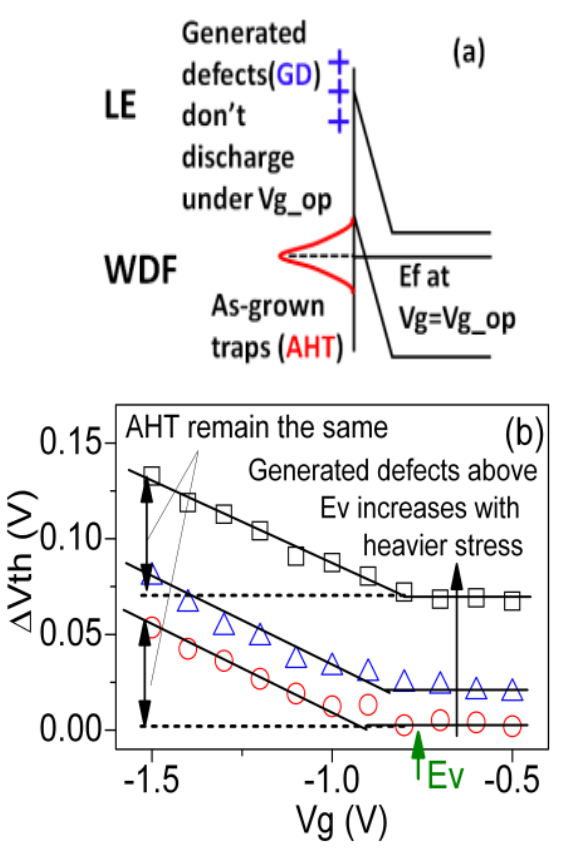

Fig. 1 (a) A schematic illustration of the energy location of two groups of defects: AHTs and GDs. (b) The energy distribution of defects at different stress time: GDs above Ev increases with stress time, but AHTs do not. 


\section{DEFECTS RESPONSIBLE FOR RTN}

The conventional RTN is measured from the drain current, Id, switching between two discrete levels under a constant gate bias, $\mathrm{Vg}$, and one example is given in Fig. 2(a). When there are more than 3 traps in a device, however, the step-like Id switching generally is replaced by a complex within-a-device-fluctuation (WDF) [12], as shown in Fig. 2(b). To measure RTN/WDF, an MOSFET must be turned on. For pMOSFETs, the Fermi-level, Ef, is below Ev when turned on [11].
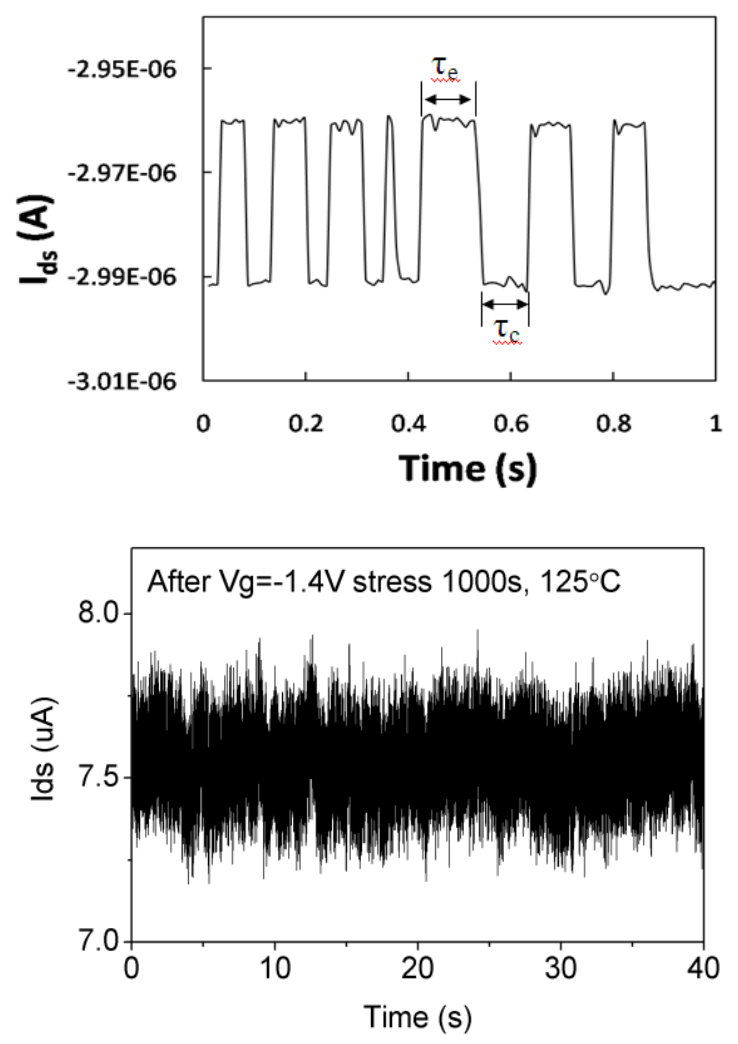

Fig. 2 Examples of RTN (a) and within-a-device -fluctuation $(W D F)(b)$.

For a defect to cause an Id fluctuation, it must be able to both charge and discharge during the measurement time window. Only traps sufficiently close to Ef satisfy this requirement. Since Ef is below Ev and AHTs are blow Ev, RTN/WDF must be caused by the AHTs according to the AG model, as illustrated in Fig. 1(a).

To support that RTN/WDF originates from asgrown defects, we examine their dependence on stress time. Fig. 3(a) shows that WDF apparently increases with stress time. This is, however, an artifact. Fig. 3(b) shows that the WDF is independent of stress time, once the measurement window is fixed, confirming that GDs do not contribute to WDF. Fig. 4 shows that an increase of measurement window enhances WDF. As a result, the increase in WDF is Fig. 3(a) originates from an increase of measurement window.
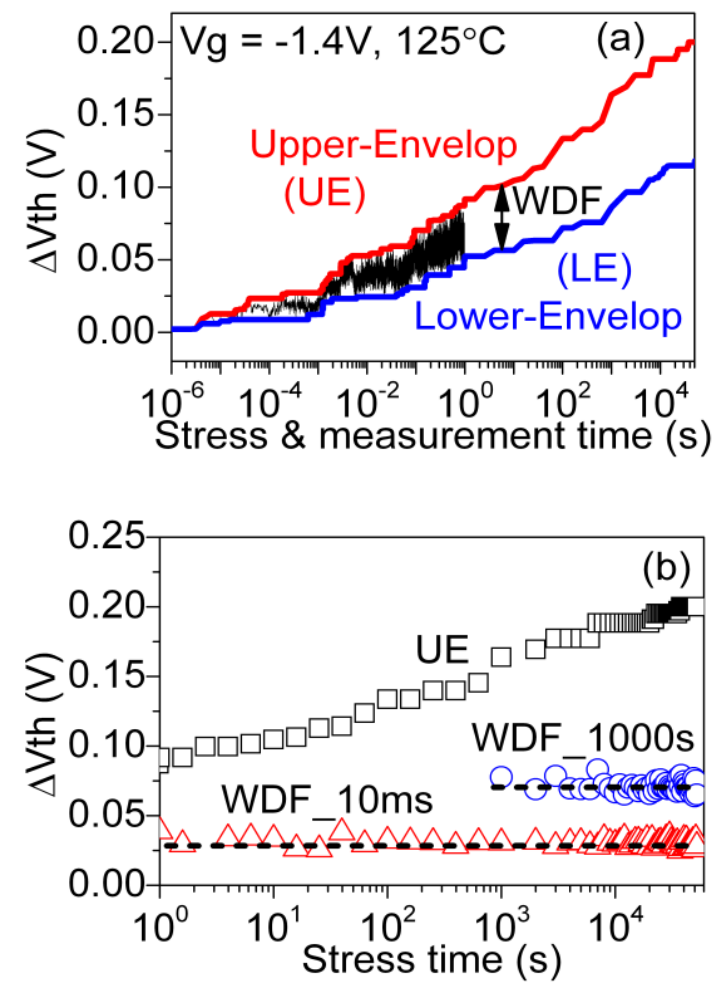

Fig. 3 (a) The $\Delta V t$ th measured continuously under $V g=$ $1.4 \mathrm{~V}$. Apart from the WDF, there are defects that do not discharge under a given $V g$, giving rise to the lower envelope, 'LE'. The upper-envelope, 'UE', equals to $W D F+L E$. The raw data also are presented for an initial 1 sec. (b) The WDF measured at a fixed time window of $10 \mathrm{~ms}$ and $1000 \mathrm{sec}$, taken at different stress time, does not increase with stress time. Even with tw $=1000 \mathrm{sec}$, WDF substantially underestimates $U E$.

\section{DEFECTS RESPONSIBLE FOR NBTI}

The NBTI is typically monitored by a shift of the threshold voltage, $\Delta$ Vth [3]. When measured at sufficiently fast speed, recovery is suppressed [10], so that both the AHTs and GDs contribute to NBTI.

Fig. 5 shows the relative contribution of AHTs (symbol ' $\square$ ') and GD ('o') to NBTI. It is clear that NBTI is dominated by AHTs initially, so that the same group of defects are responsible for both RTN/WDF and NBTI at the early stage of stress. For longer stress time, the filling of AHTs saturates, but GDs increase. As a result, GDs become increasingly important as the aging progresses.

Because of their higher energy location, GDs do not discharge during RTN/WDF measurement, so that they make no contribution to RTN/WDF. As a result, GDs cannot be detected from the RTN/WDF measurements. 


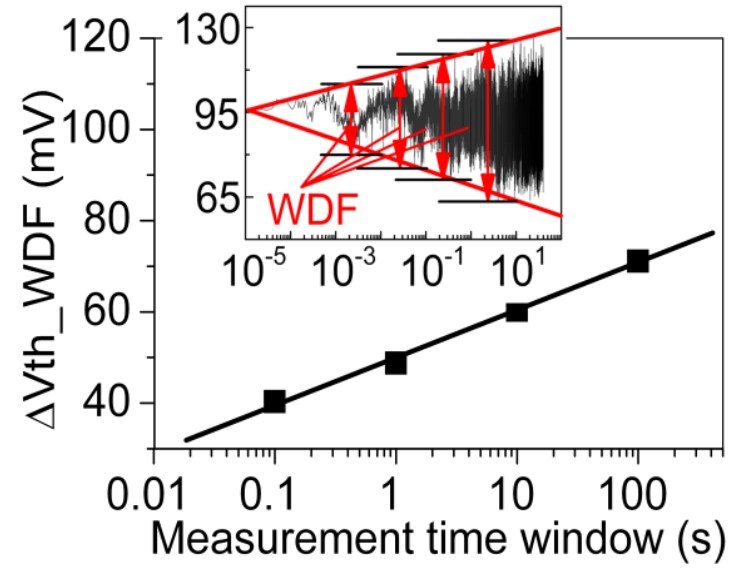

Fig. 4 Increase of WDF with the time window, tw. This device was stressed first for 1000 sec under $V g=-1.4 \mathrm{~V}$ and $125{ }^{\circ} \mathrm{C}$ to ensure that there is little changes in the number of defects during the subsequent measurement. A larger tw allows capturing slower traps, leading to the increase of WDF.

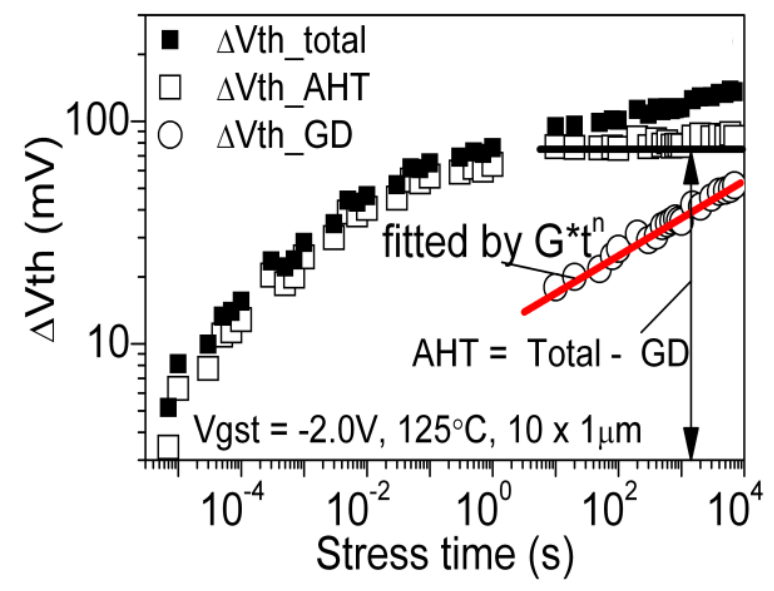

Fig. 5 The As-grown-Generation (AG) model for a devices $(W \times L=10 \times 1 \mu \mathrm{m}): \Delta V t h=A+G t^{n}$. The filling of asgrown hole trap saturates and ' $A$ ' $=$ constant for $>\sim 1$ sec.

\section{CONCLUSIONS}

In this work, we investigated the relation between RTN and NBTI through examining the defects responsible for them. By following the AG model, we show that RTN originates from the as-grown hole traps (AHTs) only, which is below silicon Ev. AHTs also dominate NBTI initially. As stress time increases, new defects are generated above silicon Ev and their relative contribution to NBTI increases. These generated defects, however, will not contribute to RTN/WDF, since they do not discharge during typical RTN/WDF measurements.

\section{ACKNOWLEDGEMENTS}

The authors thank B. Kaczer and G. Groeseneken of IMEC, Belgium, for supply of test samples used in this work. This work was supported by the EPSRC of UK under the grant no. EP/L010607/1.

\section{REFERENCES}

[1] B. Kaczer, T. Grasser, P. J. Roussel, J. Franco, R. Degraeve, L. A. Ragnarsson, E. Simoen, G. Groeseneken, and H. Reisinger, Proc. Int. Rel. Phys. Symp., 2010, pp. 26-32.

[2] P. Ren, R. Wang, Z. Ji, P. Hao, X. Jiang, S. Guo, M. Luo, M. Duan, J. F. Zhang, J. Wang, J. Liu, W. Bu, J. Wu, W. Wong, S. Yu, H. Wu, S. W. Lee, N. Xu, and R. Huang, Proc. IEDM, 2014, pp.816-819.

[3] J. F. Zhang, Microelectron. Eng., vol. 86, 2009, pp.1883-1887.

[4] D. R . Young, E. A. Irene, D. J. DiMaria, R. F. De Keersmaecker, and H. Z. Massoud, J. Appl. Phys., vol. 50, 1979, pp. 6366-6372.

[5] M. H. Chang and J. F. Zhang, J. Appl. Phys., vol.101, 2007, art.no.024516.

[6] Z. Ji, L. Lin, J. F. Zhang, B. Kaczer, and G. Groeseneken, IEEE Trans. Electron Dev., vol. 57, 2010, pp. 228-237.

[7] Z. Ji, S. F. W. M. Hatta, J. F. Zhang, J. G. Ma, W. Zhang, N. Soin, B. Kaczer, S. De Gendt, and G. Groeseneken Proc. IEDM, 2013, pp.413-416.

[8] Z. Ji, J. F. Zhang, M. H. Chang, B. Kaczer, and G. Groeseneken, IEEE Trans. Electron Dev., vol. 56, 2009, pp. 1086-1093.

[9] M. Duan, J. F. Zhang, Z. Ji, J. G. Ma, W. Zhang, B. Kaczer, T. Schram, R. Ritzenthaler, G. Groeseneken, and A. Asenov, Proc. IEDM, 2013, pp. 774-777, 2013.

[10] . L. Lin, Z. Ji, J. F. Zhang, W. D. Zhang, B. Kaczer, S. De Gendt, and G. Groeseneken, IEEE Trans. Electron Dev., vol. 58, 2011, pp. 1490-1498.

[11] S. F. W. M. Hatta, Z. Ji, J. F. Zhang, M. Duan, W. Zhang, N. Soin, B. Kaczer, S. De Gendt, and G. Groeseneken, IEEE Trans. Electron Dev., Vol. 60, 2013, pp. 1745-1753.

[12] M. Duan, J. F. Zhang, Z. Ji, W. Zhang, B. Kaczer, T. Schram, R. Ritzenthaler, G. Groeseneken, and A. Asenov, IEEE Trans. Electron Dev., Vol. 60, 2013, pp. 2505-2511. 